ORIGINAL ARTICLE

\title{
A multivariate "time based" analysis of SIDS risk factors
}

\author{
T Matthews, M McDonnell, C McGarvey, G Loftus, M O'Regan
}

Arch Dis Child 2004;89:267-271. doi: 10.1136/adc.2002.025486

See end of article for authors' affiliations

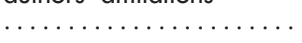

Correspondence to: Prof. T Matthews, The Children's University Hospital, Temple St, Dublin 1, Republic of Ireland; tommatt@iol.ie

Accepted 8 April 2003

\begin{abstract}
Aims: To investigate the influence of analytical design on the variability of published results in studies of sudden infant death syndrome (SIDS).

Methods: The results of a prospective case-control study, of 203 cases of SIDS, and 622 control infants are presented. All variables significant on univariate analysis were included in a multivariate model analysed in nine stages, starting with sociodemographic variables, then sequentially and cumulatively adding variables relating to pregnancy history, current pregnancy, birth, the interval from birth to the week prior to death, the last week, the last 48 hours, and the last sleep period. A ninth stage was created by adding placed to sleep prone for the last sleep period.

Results: As additional variables are added, previously published SIDS risk factors emerged such as social deprivation, young maternal age, $\geqslant 3$ previous live births, maternal smoking and drinking, urinary tract infection in pregnancy, reduced birth weight, and the infant having an illness, regurgitation, being sweaty, or a history of crying/colic in the interval from birth to the week before death, with co-sleeping and the lack of regular soother use important in the last sleep period. As the model progressed through stages 1-9, many significant variables became non-significant (social deprivation, young maternal age, maternal smoking and drinking) and in stage 9 the addition of placed to sleep prone for the last sleep period caused $\geqslant 3$ previous live births and a reduced birth weight to become significant.

Conclusion: The variables found to be significant in a case-control study, depend on what is included in a multivariate model.
\end{abstract}

$\mathrm{T}$ here is considerable interest in developing infant care guidelines for parents following the success of the "back to sleep" campaigns urging the adoption of the back sleeping position for infants and the associated reduction in sudden infant death syndrome (SIDS) rates in many western countries. ${ }^{12}$ While there has been a consistent association between the prone sleeping position, cigarette smoking, and overheating/over-wrapping and an increased risk of SIDS, ${ }^{34}$ other associations have been less consistent. Contradictory, or complex, messages have been published relating to a variety of parenting practices including co-sleeping, parental alcohol use, soother use, the use of "unsafe" sleep surfaces, allowing infants heads to be covered, parental tiredness, recent respiratory/viral illness, and SIDS. ${ }^{4-7}$ A recent study found that while there was not an increased risk of SIDS at weekends, a highly significant weekend excess emerged when SIDS rates were linked to poor maternal educational achievement. ${ }^{8}$ While population differences may explain some of this heterogeneity, differences in analytical methodology need to be considered also. ${ }^{9}$ The purposes of this study were to identify variables increasing the risk of SIDS in an Irish population and to show the effect on significant variables of including other variables in the analysis.

\section{METHODS}

The data presented are the results of a five year prospective case-control study of infants dying from SIDS in the Republic of Ireland between January 1994 and December 1998. Sudden infant death syndrome was defined as the sudden death of an infant which was unexpected by history and where a thorough postmortem examination failed to show an adequate cause of death. The diagnosis of SIDS was accepted when this diagnosis was used on the death certificate. A postmortem examination is mandatory in all cases of sudden unexpected death in Ireland with the coroner directing an experienced pathologist to conduct a postmortem examination to establish a cause of death. A control group of families was picked randomly from the birth register with matching for the date of birth and geographical location of the SIDS case. Four control infants were picked for each SIDS case and both groups were invited by letter to participate in a home interview to collect information on sociodemographics, pregnancy, the infant's medical history, the home environment, current parenting practice, and details of the events surrounding the infants last 48 hours, last sleep period, and death in the SIDS cases. The parents were interviewed in their homes within six weeks of their infant's death (the average age of cases at time of death was 15.7 weeks); the average age of control infants at interview was 21.6 weeks. Death certificates were made available by the central statistics office, facilitated by the Department of Health and Children, allowing complete ascertainment of cases.

Conditional logistic regression was used, to investigate differences between cases and controls with respect to a number of potential risk factors using the statistical package Stata version 6 . Odds ratios, 95\% confidence intervals, and $\mathrm{p}$ values were calculated to express results and are presented in table 1. A temporal multivariate model of analysis was carried out using an additive stepped approach. Variables were grouped into time determined stages starting with prepregnancy variables (stage 1), and progressing through history of prior pregnancies (stage 2), variables related to the current pregnancy (stage 3), birth related variables (stage 4 ), post-delivery issues (stage 5), the week prior to death (stage 6), the 48 hours before death (stage 7), and the last sleep period (stage 8 ). A ninth stage was created by adding the additional variable of infants placed prone in the last sleep period. Each variable was introduced into the analysis in the sequence in which it was likely to occur. All variables

Abbreviations: SIDS, sudden infant death syndrome; UTI, urinary tract infection 


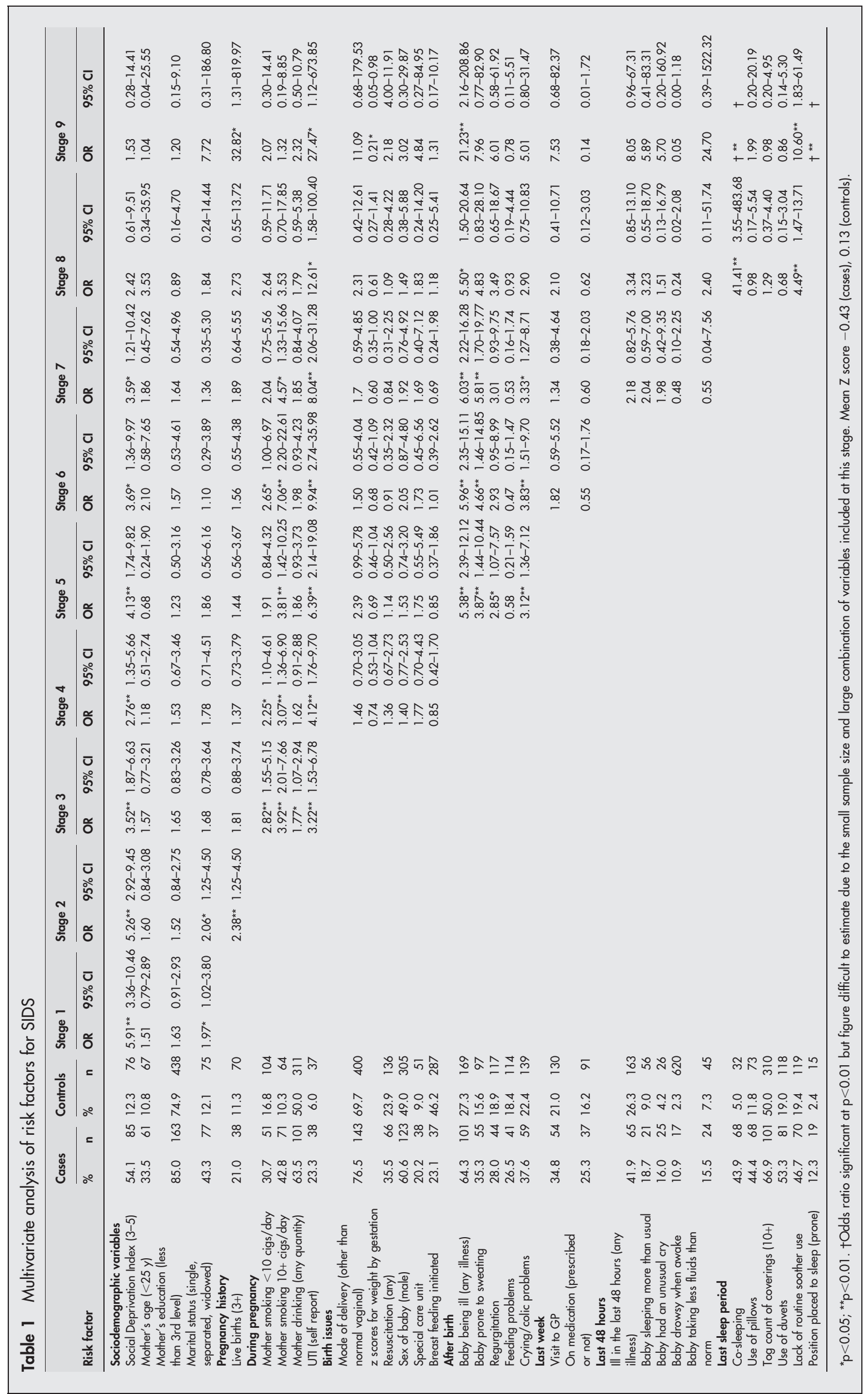


included in the multivariate analysis were significant at the univariate level, except medication in the week prior to death which we felt was an important marker of potential illness prior to death. All variables significant in univariate analysis were included in the multivariate model. The odds ratios, and associated confidence intervals and standard errors, obtained for a particular variable in a multiple logistic regression, are dependent on the other variables included in the model. A high correlation between two variables will result in substantial co-linearity problems in a multivariate model which will in turn result in an unstable outcome. The large standard errors achieved as a result along with small sample sizes for some variables may render some potentially important variables non-significant. The presentation of results in a stepwise fashion avoids masking the importance of individual variables which may occur when only the full complement of variables is presented (stage 9). The data set was also analysed using an alternative stepwise deletion procedure which involved the removal of non-significant variables. Variables were removed singly from the model, starting with the least significant-that is, the one with the highest $\mathrm{p}$ value (providing it was not less than 0.05 ), until all remaining variables were significant at $p<0.05$. The collection of variables identified as being statistically important using this alternative approach were the same as those derived in the method presented in the paper.

A social disadvantage index, scoring 0-5 (5, most deprived), was devised by adding a score of 1 for each of the following: having a medical card (a low income based free health service entitlement), being in public rented accommodation, not having a car, household unemployment (both parents unemployed), and mother on social welfare. The $\mathrm{z}$ scores for weight by gestation variable (multiples of standard deviations from the normal mean) was created by relating the birth weight of each baby to controls of similar gestation and included as a continuous variable. Computer generated norms compiled in the UK were used since norms for Ireland were not available and were adjusted for the effects of gender and parity on birth weight. ${ }^{10}$ The section on "after birth" issues are yes/no answers to open ended questions: was your baby ill, prone to sweating or regurgitation, feeding problems, or problems with crying or colic. In the "last week" section medication refers to any medication usage, both prescribed and non-prescribed. Co-sleeping was defined as an infant sharing a bed/sofa/armchair with parent(s)/relative). Data were not provided for every variable in each case and this accounts for the variation in proportions of subjects from one variable to the next. Data may be accepted as missing at random.

\section{RESULTS}

A total of 203 SIDS cases and 622 control infants formed this study. From a total of 250 SIDS families, $81 \%$ of parents agreed to take part in the study. An average of three control families per case were included, and agreed to participate, resulting in a response rate of $77 \%$. Table 1 presents the results. In stage 1 (sociodemographic variables), although maternal age, marital status (single, separated, or widowed) and education (lack of 3rd level) were significant at univariate level, only maternal marital status remains significant at the multivariate level. Social disadvantage remains significant when maternal age, marital status, and education are controlled for, with those parents scoring 3-5 (most deprived) being six times more likely to have SIDS cases compared to those scoring $0-2$ (most advantaged) in the social disadvantage index.

In stage 2, pregnancy history is added to the model with social disadvantage and maternal marital status remaining significant. Mothers with three or more previous live births are 2.38 times more likely to have SIDS cases than those who had less than three previous live births.

In stage 3, current pregnancy related variables are added to the model, and while social deprivation remains significant, the odds ratio is reduced from 5.26 to 3.52 and maternal marital status is no longer a significant factor. All of the previously significant pregnancy related variables remain significant when previous history and social demographic variables are controlled for. Mothers who smoke $<10$ cigarettes per day are 2.82 times more likely than nonsmokers to have SIDS cases, while those who smoke 10 or more cigarettes per day are four times more likely than nonsmokers to have SIDS cases. Both maternal alcohol consumption (OR 1.77) and a self reported history of urinary tract infection during pregnancy (OR 3.22) are significant.

In stage 4, birth related issues are added and maternal smoking, social disadvantage, and urinary tract infection (UTI) in pregnancy remain significant; however, maternal drinking during pregnancy is no longer significant. The odds ratio for social disadvantage is reduced to 2.76. None of the six variables introduced at this stage are significant when adjusted for the effects of sociodemographic and pregnancy related variables.

Adding postnatal variables (stage 5) shows social disadvantage, maternal smoking ( $>10$ cigarettes per day), and UTI in pregnancy to be significant variables, with smoking $<10$ cigarettes losing significance. UTI in pregnancy (OR 6.39 ) and social disadvantage (OR 4.13) remain significant. Other significant variables are the baby being ill (OR 5.38), prone to sweating (OR 3.87), regurgitation (OR 2.85), and experiencing problems with crying/colic (OR 3.12).

In stage 6, social disadvantage, maternal smoking (both $<10$, and $10+$ with a dose-response effect) UTI during pregnancy, the baby being ill, prone to sweating, and having crying/colic problems but not regurgitation, remain significant, although a visit to the GP, or medication usage were not. The strength of the association between UTI in pregnancy and SIDS is increased further by the addition of these variables (OR 9.94).

In stage 7 , none of the newly added variables achieved significance. The previously significant risk factors remain so at this stage of the analysis with the exception of maternal smoking $<10$ cigarettes per day. The odds ratio for UTI in pregnancy is now reduced to 8.04 and that of maternal smoking more than 10 cigarettes per day is reduced from 7.15 to 4.57. Social disadvantage is unchanged (OR 3.59, from 3.69) whilst the odds ratio for baby prone to sweating is increased from 4.66 to5.81.

In stage 8, variables relating to the infant's last sleep period are added and result in the removal of the previously established significance for social disadvantage, maternal smoking of 10 or more cigarettes per day, and infants with crying or colic problems or being prone to sweating. However, the infant being ill from birth to the week before death (OR 5.50) and maternal UTI in pregnancy (OR 12.61) remain significant. The use of pillows, duvets, or a total clothing and bedding tog score of greater than 10 are not significant at this stage. Co-sleeping was a significant risk factor when all other variables listed are controlled for with an odds ratio of 41.41. In a univariate analysis the odds ratio associated with cosleeping was 22.93 (95\% CI 10.93 to 48.08 ; p $<0.001$ ). Soother use was a complex variable with univariate analysis of the data, suggesting that usual use of a soother was a significant risk for SIDS (OR 1.95; 95\% CI 1.25 to 3.06; p<0.01) while use of a soother in the last sleep period emerged as being a protective factor (OR 0.33; 95\% CI 0.22 to 0.50 ; $\mathrm{p}<0.001$ ). Further examination of the data showed that while $77 \%$ of cases habitually used a soother, only $30 \%$ did so on the night of the last sleep. Thus the absence of routine soother use in 
the last sleep period was used in the multivariate analysis and remained a significant risk factor with an odds ratio of 4.49 .

In stage 9, the single variable of infants placed prone to sleep for the last sleep period was added. This odds ratio for this variable was statistically significant $(p<0.001)$ in multivariate analysis, but difficult to calculate due to the small proportion of babies in total (34 of 825) who were placed in the prone position for their last sleep along with the large combination of variables included in the analysis. In a univariate analysis use of the prone position had an odds ratio of 7.88 (95\% CI 3.09 to $20.11, \mathrm{p}<0.001$ ). Other factors which remained significant were UTI in pregnancy (OR 27.47), baby being ill anytime from birth to the week before death (OR 21.23), and lack of soother use (OR 10.62). In addition, having at least three previous live births becomes significant again at this stage (OR 32.82), having last been significant in stage 2 , and a reduced birth weight for gestation appears for the first time as a significant variable (OR 0.21 for high z scores).

\section{DISCUSSION}

The recent "back to sleep" campaigns, where parents were advised to avoid epidemiologically derived SIDS risk factors, namely the prone sleeping position, overheating, overwrapping, and parental smoking, have been associated with a dramatic and sustained fall in SIDS rates in all western countries mounting such campaigns. ${ }^{4}$ This has been hailed as one of the great public health triumphs of the modern era with thousands of infant deaths prevented annually worldwide. In Ireland a similar campaign saw the SIDS rate fall from 2.1/1000 live births (1980-90 inclusive) to 0.7-0.8 for the years 1994-2000 inclusive, with 70-80 less infant deaths per year. ${ }^{11}$ This has given impetus to the continued analysis, and reanalysis, of case-control databases to confirm the validity of established SIDS risk factors, both within and across national boundaries, and to see if new undiscovered risk factors will allow further simple risk reduction measures. However, a recent review of the epidemiological literature relating to SIDS clearly documents the variability in the published results. ${ }^{4}$ Meta-analysis has been used to develop pooled odds ratios across different studies in an attempt to overcome these difficulties. ${ }^{12}$ However, a recent review of the causes for this heterogeneity in the epidemiology of SIDS concluded that the use of meta-analysis could have minimised the importance of prone sleeping in SIDS. ${ }^{9}$

As an example of the difficulty in developing consistent messages for parents, professionals, and health agencies, many studies have shown increased SIDS rates for disadvantaged minority groups within countries, an association which disappears in studies controlling for maternal education, family income, and use of the prone sleeping position. $^{13}{ }^{14}$ Similarly the role of viral/respiratory infection in SIDS has been debated for many years with several recent studies finding no association. ${ }^{3}{ }^{15}$ However, other studies have shown an association between intercurrent infection and SIDS in infants who are sleeping prone, ${ }^{16}$ or are heavily wrapped while sleeping. ${ }^{17}$ In the present study we found that the baby having any illness from birth to the week prior to death remained a significant risk factor throughout the analysis, whereas in the 48 hours prior to death the presence of illness, a visit to a family doctor, or medication use were not significant, even when controlling for the amount of clothing or bedding used and for the prone sleeping position. If this is a true reflection of the situation it means that interventions aimed at improving parental recognition of illness immediately prior to death are likely to be fruitless, and blaming parents for missing signs of illness immediately prior to death (both common practices) are unjustified.
Interestingly a maternal urinary tract infection in pregnancy (self report, not confirmed from urine culture results) remained a very powerful and consistent risk factor throughout the sequential analysis as presented. Maternal UTI in pregnancy has long been found to be a significant risk factor for SIDS, forming part of the high risk scoring system devised and used in Sheffield (UK) in the 1970s and 1980s. ${ }^{18}$ Maternal UTI in pregnancy has been associated with an increased risk of premature delivery. ${ }^{19} 20$ If this epidemiological association is true, then it lends further support to the concept of SIDS infants being predisposed or vulnerable to sudden death by an adverse intrauterine environment. ${ }^{21}$

A benefit of conducting the analysis in the manner presented in this paper is the clear and obvious effect the addition of further variables has on both previously derived levels of significance, or in some cases as to whether a variable remains significant or not. In this paper variables found to significantly increase the risk of SIDS include social deprivation, at least three previous live births, maternal cigarette smoking and drinking (alcohol) during pregnancy, a slightly reduced birth weight, a parental report of the baby being ill or experiencing crying/colic problems in the interval from birth to the week prior to death/interview (controls), and in the last sleep period either being placed prone to sleep, co-sleeping, or not using a soother. ${ }^{4}$ However, as the analysis progressed through stages 1-9 many variables changed their level of and ultimately lost their significance. Some studies in the literature control for few confounding factors, presenting simple unadjusted odds ratios, while others control for the combinations of variables found in any one of the nine stages of analysis presented in this paper. An example of the most worrying aspect of this degree of statistical complexity is the ability to remove smoking as a significant risk factor for SIDS by the addition of the variables relating to the last sleep period (stage 8). The weight of data linking maternal smoking to SIDS is overwhelming, with a meta-analysis of over 50 articles confirming an increased risk, most showing a clear dose-response effect, as is this paper in stages 3, 4, and 6 , causing some authors to suggest a causal relation. ${ }^{12}$

While statisticians have always appreciated the amount of statistical manoeuvring possible in large epidemiological databases, the degree of fine tuning of results possible has perhaps been underestimated by those of us who are statistically naive in both the medical and wider communities. Case-control studies have proved hugely beneficial in medicine, and recently in reducing SIDS rates despite the limitations already discussed. Because of the importance of the messages derived from such studies to modern medical practice, perhaps it is time to revisit the methods used to analyse large case-control databases.

\section{ACKNOWLEDGEMENTS}

The Irish National Sudden Infant Death Register is funded by the Irish Dept of Health and Children, and is owned by the Irish Sudden Infant Death Association, a voluntary parents group.
Authors' affiliations
T Matthews, University College Dublin, Dept of Paediatrics, The Children's University Hospital, Temple St, Dublin, Republic of Ireland M McDonnell, C McGarvey, G Loftus, The National Sudden Infant Death Register, The Children's Hospital, Temple St, Dublin, Republic of Ireland M O'Regan, Dept of Statistics, Trinity College, Dublin, Republic of Ireland

\section{REFERENCES}
1 Dwyer T, Ponsonby A-L. The decline of SIDS: a success story for epidemiology. Epidemiology 1996;7:323-5.
2 Wennergren G, Alm B, Oyen N, on behalf of the Nordic Epidemiologic SIDS Study, et al. The decline in the incidence of SIDS in Scandinavia and its relation to risk intervention campaigns. Acta Paediatr 1997;86:963-8. 
3 Brook H, Gibson A, Tappin D, et al. Case control study of sudden infant death syndrome in Scotland, 1992-5. BMJ 1997;314:1516-20.

4 Hauk F. The changing epidemiology of SIDS. In: Byard RW, Krous HF, eds. Sudden infant death syndrome, problems, progress and possibilities. London: Arnold, 2001:31-57.

5 Blair PS, Fleming PJ, Bensley D, et al. Smoking and the sudden infant death syndrome: results from 1993-5 case-control inquiry into stillbirths and deaths in infancy. BMJ 1996:313:195-8.

6 L'Hoir MP, Engelberts AC, van Well GTHJ, et al. Case control study of current validity of previously described risk factors for SIDS in the Netherlands. Arch Dis Child 1998;79:386-93.

7 Alm B, Wennergren G, Norvenius G, et al, on behalf of the Nordic Epidemiological SIDS Study. Caffeine and alcohol as risk factors for sudden infant death syndrome. Arch Dis Child 1998;81:107-11.

8 Spiers PS, Guntheroth WG. The effect of the weekend on the risk of sudden infant death syndrome. Pediatrics 1999;104:11119-20.

9 Dwyer T, Couper D, Walter SD. Sources of heterogeneity in the meta-analysis of observational studies: the example of SIDS and sleeping position. J Clin Epidemiol 2001;54:440-7.

10 Tin W, Wariyar UK, Hey EN. Selection biases invalidate current low birth weight-for-gestation standards. Br J Obstet Gynaecol 1997;104:180-5.

11 Mehanni M, Cullen A, Kiberd B, et al. The current epidemiology of SIDS in Ireland. Ir Med J 2000;93:264-8.

12 Mitchell EA, Milerad J. Smoking and sudden infant death syndrome. In: International consultation on environmental tobacco smoke (ETS) and child health. Geneva: World Health Organisation, 1999:105-29.
13 Mitchell EA, Tuohy PG, Brunt JM, et al. Risk factors for sudden infant death syndrome following the prevention campaign in New Zealand; a prospective study. Pediatrics 1997; 100:835-40.

14 Kraus JF, Greenland S, Bulterys M. Risk factors for sudden infant death syndrome in the US Collaborative Perinatal Project. Int J Epidemiol 1989;18:113-20.

15 Hoffman HJ, Damus K, Hillman L, et al. Risk factors for SIDS: results of the National Institute of Child Health and Human Development SIDS cooperative Epidemiological study. Ann N Y Acad Sci 1988;533:13-30.

16 Ponsonby A-L, Dwyer T, Gibbons LE, et al. Factors potentiating the risk of sudden infant death syndrome associated with the prone position. N Engl J Med 1993;329:377-82.

17 Gilbert R, Rudd P, Berry J, et al. Combined effect of infection and heavy wrapping on the risk of sudden unexpected infant death. Arch Dis Child 1992;67:171-7.

18 Carpenter RG, Gardner A, McWeeny PM, et al. Multistage scoring system for identifying infants at risk of unexpected death. Arch Dis Child 1977:52:606-12.

19 Schieve LA, Handler A, Hershow R, et al. Urinary tract infection during pregnancy: its association with maternal morbidity and perinatal outcome. Am J Public Health 1994;84:405-10.

20 Leviton A, Paneth N, Lynne Reuss $M$, et al. Maternal infection, fetal inflammatory response, and brain damage in very low birth weight infants. Pediatr Res 1999;46:566-75.

21 Kinney H, Brady BA, Finkelstein DM, et al. Delayed central nervous system myelination in the sudden infant death syndrome. J Neuropathol Exp Neurol 1991;50:29-48. 